Classification

Physics Abstracts

$42.55 \mathrm{~N}-42.60 \mathrm{~B}$

\title{
Erbium Laser Model for Two-Wavelength Operation
}

François Sanchez $\left({ }^{1}\right)$, Marc Le Flohic $\left({ }^{1}\right)$, Pascal Besnard $\left({ }^{1}\right)$, Pierre-Luc François $\left({ }^{2}\right)$ and Guy Michel Stephan $\left({ }^{1}\right)$

(1) ENSSAT, Laboratoire d'Optronique, équipe associée au CNRS (EP0001), 6 rue de Kérampont, B.P. 447, 22305 Lannion Cedex, France

(2) France Telecom, CNET, Lannion B, route de Trégastel, 22300 Lannion Cedex, France

(Received 25 April 1994, revised 25 October 1994, accepted 9 December 1994)

\begin{abstract}
A theoretical modeling of dual wavelength operation of erbium-doped fibre lasers is presented. The model is a generalization of the one used in the single mode theory, based on the existence of isolated ions and ion pairs coupled via the laser field. Analytical results are obtained in the steady state. The linear stability analysis of the coupled equations shows the existence of two pairs of complex conjugate eigenvalues and two real negative eigenvalues. These results imply that the system is driving by two frequencies : one associated with the classical relaxation oscillation and a low frequency characteristic of a bimodal system. When the ion pair concentration is increased, the real part of one pair of conjugate eigenvalues becomes positive. In such a situation, self-pulsing and chaotic dynamics occur together with antiphase effects between two mode intensities. Locking of the low frequency on a subharmonic of the high frequency is also obtained leading to $n T$-periodic orbits.
\end{abstract}

\section{Introduction}

Multimode lasers are a fascinating field of investigation due to their various and complex dynamics. Among them antiphase, or winner takes all, dynamics have recently received great attention [1-3]. In this context, rare-earth doped fibre lasers are of particular interest because of their large variety of dynamical behaviors [4-6].

Erbium-doped fibre lasers (EDFL) can operate spontaneously either in a c.w. or a selfpulsing regime when lasing around $1.55 \mu \mathrm{m}$, depending on both the ion pair concentration $x$ and the pumping rate [7]. Indeed for $x \approx 10 \%$ EDFL are self-pulsing for pumping rates ranging from the threshold to a higher value beyond which the laser becomes c.w. A simple theoretical modeling of the dynamics has been proposed in [8]. The model is based on the existence of isolated ions and ion pairs coupled with the laser field. We have found that when EDFLs having a pair concentration $x=18 \%$ operate simultaneously at $1.55 \mu \mathrm{m}$ and $1.536 \mu \mathrm{m}$, the laser exhibits antiphase effects between the two wavelength intensities and chaotic dynamics [6]. Moreover, we have reported a quasi-periodic route to chaos for a fibre having an ion pair concentration of $x=7.5 \%$ operating with dual wavelengths [9]. A sixdimensional model has been numerically solved which gives good agreement with the experimental data.

In this paper, we investigate the model proposed in [9]. In Section 2 we present the model and transform it into a suitable form allowing the derivation of the bimode steady state. This latter is calculated in Section 3 where the evolution of the intensities versus the pumping 
ratio is investigated. Section 4 is devoted to the linear stability analysis of the coupled equations. In particular it is shown that the system is driving by two frequencies and that, depending on the $x$ value, the steady state is unstable over some range of pumping rates. The coupled equations are numerically solved in Section 5. Antiphase effects are obtained in both the transient regime leading towards a stable c.w. state and the $n T$-periodic regimes for unstable steady states. The chaotic regime is analysed.

\section{Theoretical Modeling}

A simple model of erbium-doped fibre laser for single mode operation has been developed in [8]. In this paper we propose and investigate an improved model for dual wavelength operation. From the beginning, it should be clear that we are modeling the relaxed multimode laser by two supermodes or clusters of modes respectively centered around 1.55 and $1.536 \mu \mathrm{m}$ [5, 9]. In [6] we have reported antiphase dynamics and chaos for two-wavelength operation of an EDFL with an ion pair concentration of $x=18 \%$. The antiphase states correspond, in our work, to the two wavelength intensities. In particular, we have observed a chaotic regime in which periodic windows appear. In [9], the dynamics of an EDFL with $x=7.5 \%$ has been studied in the same experimental configuration. In this case, starting from a c.w. state for high pumping rates we have identified a quasi-periodic route to chaos when the pumping ratio is decreased. The typical scenario is : c.w. $\rightarrow T$-periodic $\rightarrow 2 T$ periodic $\rightarrow 3 T$-periodic $\rightarrow$ chaos $\rightarrow T$-periodic. Antiphase dynamics occur in the $n T$-periodic and chaotic regimes. A low frequency spectra analysis reveals the existence of two frequencies. The observed periodic regimes correspond to a frequency locking of the low frequency on a subharmonic of the high frequency. Moreover, the chaotic regime is achieved when no locking and high coupling occur between these two characteristic frequencies.

A theoretical model has been proposed in [9] for dual wavelength operation. The model was based on the well-known two-mode laser model where we have added two equations for the population dynamics of ion pairs. The analysis was, however, restricted to numerical simulation of the nonlinear coupled equations. The theoretical dynamical scenario versus $r$ was found to be the same as that observed experimentally which provides justification for the simple model used. In the following we will give the set of equations describing the dynamics of the EDFL in dual wavelength operation :

$$
\begin{aligned}
& \frac{\partial d_{1}}{\partial t}=A-a_{2}\left(1+d_{1}\right)-2 d_{1}\left(I_{1}+\beta I_{2}\right) \\
& \frac{\partial d_{2}}{\partial t}=-a_{2}\left(1+d_{2}\right)+\gamma A-2 d_{2}\left(\beta I_{1}+I_{2}\right) \\
& \frac{\partial d_{+}}{\partial t}=a_{2}\left(1-d_{+}\right)-a_{22}\left(d_{-}+d_{+}\right) / 2+\left(2-3 d_{+}\right)\left(I_{1}+I_{2}\right) y \\
& \frac{\partial d}{\partial t}=A-a_{2}\left(1-d_{+}\right)-a_{22}\left(d_{-}+d_{+}\right) / 2-d_{-}\left(I_{1}+I_{2}\right) y \\
& \frac{\partial I_{1}}{\partial t}=I_{1}\left(-1+A\left(d_{1}+\beta d_{2}\right)(1-2 x)+A d_{-} x y\right) \\
& \frac{\partial I_{2}}{\partial t}=I_{2}\left(-1+A\left(d_{2}+\beta d_{1}\right)(1-2 x)+A d_{-} x y\right)
\end{aligned}
$$


where $I_{1,2}$ are the normalised laser intensities, $d_{1,2}$ the corresponding population inversions for isolated (amplifying) ions and $d_{ \pm}=n_{22} \pm n_{11}$ are associated with ion pairs (saturable absorber). $A$ represents the pumping rate, $x$ the ion pair concentration, $y$ is the ratio between the absorption cross-sections of an ion pair and an isolated ion, $n_{\mathrm{k}}$ is the population of level $|\mathrm{k}\rangle$ for the isolated ions and $n_{\mathrm{kl}}$ is the population of level $|\mathbf{k l}\rangle$ for the ion pairs. $a_{2}=\tau_{1} / \tau_{2}$, $a_{22}=\tau_{1} / \tau_{22}, A=\sigma_{1} N_{0} \tau_{1}, N_{0}$ is the erbium concentration, $\tau_{1}$ is the photon lifetime and $\tau_{\mathrm{k}}$ is the lifetime of level $|\mathrm{k}\rangle . \beta$ is the cross-saturation parameter and $\gamma$ represents a dichroism in the pumping process in order to take into account the small anisotropies of the laser. In the experiment the net gains for the two frequencies are different because of small anisotropies induced by twists or bends of the fibre. For convenience and simplicity, we have assumed in system or bends of the fibre. For convenience and simplicity, we have assumed in system (1) that the effect of an ion pair is the same for the two fields as well as the losses. Equations (1a) and (1b) describe the population dynamics of isolated ions, equations (1c) and (1d) the population dynamics of ion pairs and equations (1e) and (1f) the dynamics of the two fields considered here. The set of equations $(1 a, b, e, f)$ is similar to that used in [5] in the case of bi-polarized $\mathrm{Nd}$-doped fibre laser.

The notations used are illustrated in Figure 1 which shows the schematic energy diagram of an isolated ion and an ion pair. Isolated erbium ions are described as two-level systems (Fig. 1a). Ion pairs are assumed to be three-level systems (Fig. 1b) where the states $|11\rangle$, $|12\rangle,|22\rangle$, correspond respectively to two neighboring ions in the states $\left({ }^{4} \mathrm{I}_{15 / 2},{ }^{4} \mathrm{I}_{15 / 2}\right)$, $\left({ }^{4} \mathrm{I}_{15 / 2},{ }^{4} \mathrm{I}_{13 / 2}\right),\left({ }^{4} \mathrm{I}_{13 / 2},{ }^{4} \mathrm{I}_{13 / 2}\right)$. The resulting energy diagram is obtained by neglecting the interaction energy between the two ions. The short lifetime $\left(\tau_{22}\right)$ of level $|22\rangle$ results from the energy transfer between two excited neighboring ions [8].

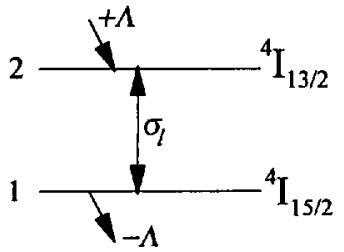

(a)

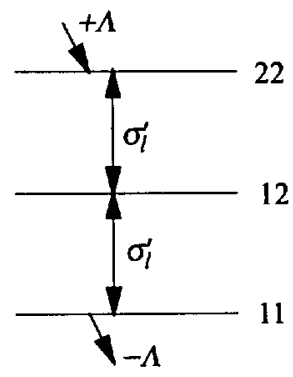

(b)

Fig. 1. - Schematic energy levels of : (a) an isolated ion and (b) an ion pair.

Let us make the following transformation for the variables :

$$
\begin{aligned}
& d_{1}=(D+d) / 2 \\
& d_{2}=(D-d) / 2 \\
& I_{1}=(I+i) / 2 \\
& I_{2}=(I-i) / 2 .
\end{aligned}
$$


Combining system (1) with transformation (2) yields :

$$
\begin{aligned}
& \frac{\partial D}{\partial t}=-2 a_{2}-a_{2} D+A(1+\gamma)-d(1-\beta) i-D(1+\beta) I \\
& \frac{\partial d}{\partial t}=-a_{2} d+A(1-\gamma)-D(1-\beta) i-d(1+\beta) I \\
& \frac{\partial d_{+}}{\partial t}=a_{2}\left(1-d_{+}\right)-a_{22}\left(d_{-}+d_{+}\right) / 2+\left(2-3 d_{+}\right) I y \\
& \frac{\partial d_{-}}{\partial t}=A-a_{2}\left(1-d_{+}\right)-a_{22}\left(d_{-}+d_{+}\right) / 2-d_{-} I y \\
& \frac{\partial I}{\partial t}=\frac{A d}{2}(1-\beta)(1-2 x) i+\left(-1+\frac{A D}{2}(1+\beta)(1-2 x)+A d_{-} x y\right) I \\
& \frac{\partial i}{\partial t}=\frac{A d}{2}(1-\beta)(1-2 x) I+\left(-1+\frac{A D}{2}(1+\beta)(1-2 x)+A d_{-} x y\right) i .
\end{aligned}
$$

System (3) is now in suitable form to allow the determination of the bimode steady state.

\section{Steady State}

In this section we are interested in obtaining the steady state values of the dynamical variables which will be used for the linear stability analysis. System (3) has three stationary states [5]: i) the off-state $\left(I_{1}=0, I_{2}=0\right)$ stable up to the laser threshold $A_{\text {th1 }}$; ii) two single-mode states $\left(I_{1} \neq 0, I_{2}=0\right)$ stable between $A_{\text {th1 }}$ and $A_{\text {th2 }}$ and $\left(I_{1}=0, I_{2} \neq 0\right)$ always unstable; iii) the bimode state $\left(I_{1} \neq 0, I_{2} \neq 0\right)$ stable beyond the second threshold $A_{\mathrm{th} 2}$. The calculation presented in this section has been performed with the help of Mathematica [10] and is restricted to the bimode stationnary state because we are mainly interested in the dynamics due to the coupling between the two modes. We first express $D, d$, $d_{+}, d_{-}$and $i$ as functions of the steady state value of $I$ and then solve an equation for $I$. At the steady state all the derivatives vanish. Above the lasing threshold (i.e. $I \neq 0$ ) system (3) becomes :

$$
\begin{aligned}
& -2 a_{2}-a_{2} \bar{D}+A(1+\gamma)-d(1-\beta) \bar{i}-\bar{D}(1+\beta) \bar{I}=0 \\
& -a_{2} \bar{d}+A(1-\gamma)-\bar{D}(1-\beta) \bar{i}-\bar{d}(1+\beta) \bar{I}=0 \\
& a_{2}\left(1-a_{+}\right)-a_{22}\left(\bar{d}_{-}+\bar{d}_{+}\right) / 2+\left(2-3 \bar{d}_{+}\right) \bar{I} y=0 \\
& A-a_{2}\left(1-\bar{d}_{+}\right)-a_{22}\left(\bar{d}_{-}+\bar{d}_{+}\right) / 2-\bar{d}_{-} \bar{I} y=0 \\
& -1+\frac{A \bar{D}}{2}(1+\beta)(1-2 x)+A \bar{d}_{-} x y=0 \\
& A(1-\beta) d(1-2 x)=0
\end{aligned}
$$


where the bar indicates the steady state values.

Equation (4f) leads to :

$$
a=0
$$

Equations (4a) and (5) give :

$$
\bar{D}=\frac{-2 a_{2}+\Lambda(1+\gamma)}{a_{2}+\bar{I}+\beta \bar{I}}
$$

Equations (4b), (5) and (6) yield :

$$
\bar{i}=\frac{\Lambda(1-\gamma)\left(a_{2}+\bar{I}+\beta \bar{I}\right)}{(-1+\beta)\left(2 a_{2}-\Lambda-\Lambda \gamma\right)}
$$

Combining (4c) and (4d) leads to :

$$
\begin{aligned}
& \bar{d}_{-}=\frac{-2 a_{2} a_{22}+2 a_{2} A+a_{22} A-2 a_{2} \bar{I} y-2 a_{22} \bar{I} y+6 A \bar{I} y}{2\left(a_{2} a_{22}+a_{2} \bar{I} y+2 a_{22} \bar{I} y+3 \bar{I}^{2} y^{2}\right)} \\
& d_{+}=\frac{2 a_{2} a_{22}-a_{22} A+2 a_{2} \bar{I} y+2 a_{22} \bar{I} y+4 \bar{I}^{2} y^{2}}{2\left(a_{2} a_{22}+a_{2} \bar{I} y+2 a_{22} \bar{I} y+3 \bar{I}^{2} y^{2}\right)}
\end{aligned}
$$

Combining expressions (6) to (9) with equation (4e) leads to a third order polynomial equation for the steady state intensity $\bar{I}$ :

$$
\begin{aligned}
& -6(1+\beta) \bar{I}^{3} y^{2}+ \\
& \bar{I}^{2} y\left[\begin{array}{c}
-2 a_{2}-4 a_{22}-2 a_{2} \beta-4 a_{22} \beta-6 a_{2} y-6 A a_{2} y-6 A a_{2} \beta y+ \\
10 A a_{2} x y-2 A a_{22} x y+10 A a_{2} \beta x y-2 A a_{22} \beta x y+3 A y \Lambda+ \\
3 A \beta y A+3 A \gamma y A+3 A \beta \gamma y A-6 A \gamma x y A-6 A \beta \gamma x y A
\end{array}\right]+ \\
& {\left[\begin{array}{c}
-2 a_{2} a_{22}-2 a 2 a_{22} \beta-2 a_{22} y-2 A a_{2}^{2} y-4 a_{2} a_{22} y-4 A a_{2} a_{22} y- \\
2 A a_{2}^{2} \beta \gamma-4 A a_{2} a_{22} \beta y+4 A a_{2}^{2} x y+6 A a_{2} a_{22} x y+4 A a_{2}^{2} \beta x y+ \\
6 A a_{2} a_{22} \beta x y-2 A a_{2}^{2} x y-2 A a_{2} a_{22} x y^{2}+A a_{2} y A+2 A a_{22} y A+ \\
A a_{2} \beta y \Lambda+2 A a_{22} \beta y \Lambda+A a_{2} \gamma y A+2 A a_{22} \gamma y A+A a_{2} \beta \gamma y \Lambda+ \\
2 A a_{22} \beta \gamma y \Lambda-3 A a_{22} x y \Lambda-3 A a_{22} \beta x y \Lambda-2 A a_{2} \gamma x y A- \\
4 A a_{22} \gamma x y A-2 A a_{2} \beta \gamma x y \Lambda-4 A a_{22} \beta \gamma x y \Lambda+6 A a_{2} x y^{2} \Lambda
\end{array}\right]+} \\
& {\left[\begin{array}{c}
-2 a_{2}^{2} a_{22}-2 A a_{2}^{2} a_{22}-2 A a_{2}^{2} a_{22} \beta+4 A a_{2}^{2} a_{22} x+4 A a_{2}^{2} a_{22} \beta x- \\
2 A a_{2}^{2} a_{22} x y+A a_{2} a_{22} A+A a_{2} a_{22} \beta A+A a_{2} a_{22} \gamma A+ \\
A a_{2} a_{22} \beta \gamma A-2 A a_{2} a_{22} x \Lambda-2 A a_{2} a_{22} \beta x \Lambda-2 A a_{2} a_{22} \gamma x A- \\
2 A a_{2} a_{22} \beta \gamma x A+2 A a_{2}^{2} x y \Lambda+A a_{2} a_{22} x y A
\end{array}\right]=0 .}
\end{aligned}
$$

The threshold is obtained from (10) by taking $I=0$ and solving for $A$ : 


$$
\begin{aligned}
& A_{t h}= \\
= & \frac{2 a_{2} a_{22}(1+A+A \beta-2 A x-2 A \beta x+A x y)}{A\left(a_{22}+a_{22} \beta+a_{22} \gamma+a_{22} \beta \gamma-2 a_{22} x-2 a_{22} \beta x-2 a_{22} \gamma x-2 a_{22} \beta \gamma x+2 a_{2} x y+a_{22} x y\right)}
\end{aligned}
$$

This expression matches those obtained in the single-mode model by taking $\gamma=1$ and $\beta=0$. In fact the threshold value obtained in this way corresponds to the extrapolation of the bimode solution for $I=0$ and is very close to the exact threshold of the single-mode state $A_{\mathrm{thl}}$. Let us define the pumping ratio as $r=\Lambda / \Lambda_{\mathrm{th}}$. For $r \geqslant 1$ equation (10) has only one positive real root. As reported in [8] for the single mode case, the threshold increases with $x$.

At this stage it is interesting to look at the evolution of the steady state intensities $\bar{I}_{1}$ and $\bar{I}_{2}$ versus $r$. These evolutions are given in Figure 2 for several values of $x$. Note that the laser efficiency decreases while $x$ increases. Moreover, due to the different gains associated with the different wavelengths, the two modes have different thresholds. The lasing threshold given in (11) is very close to the threshold of the mode with lower losses. The efficiency of the first lasing mode decreases when the second mode switches on because of the crosssaturation. Similar results have been reported experimentally in $[5,6]$. For consistency, the parameters used in this section and hereafter are the same as those used in the single mode theory [8] :

$$
\begin{gathered}
\tau_{2}=10 \mathrm{~ms}, \quad \tau_{22}=2 \mu \mathrm{s}, \tau_{1}=200 \mathrm{~ns}, N_{0}=5 \times 10^{18} \mathrm{~cm}^{-3}, \\
\sigma_{1}=1.6 \times 10^{-10} \mathrm{~cm}^{3} \mathrm{~s}^{-1}, y=0.2 .
\end{gathered}
$$

The remaining parameters are: $\beta=0.5, \gamma=0.85$.

\section{Linear Stability Analysis}

We perform here a classical linear stability analysis of system (3) around its steady state obtained from relations (5) to (10). Let us consider small variations from steady state :

$$
\delta \mathbf{X}=\left(\begin{array}{c}
\delta D \\
\delta d \\
\delta d_{+} \\
\delta d_{-} \\
\delta I \\
\delta i
\end{array}\right)=\left(\begin{array}{c}
D-\bar{D} \\
d-\bar{d} \\
d_{+}-d_{+} \\
d_{-}-d_{-} \\
I-\bar{I} \\
i-\bar{i}
\end{array}\right)
$$

These variations evolve with time according to system (3) linearized around its steady state solution :

$$
\frac{\partial \delta \mathbf{X}}{\partial t}=L \delta \mathbf{X}
$$



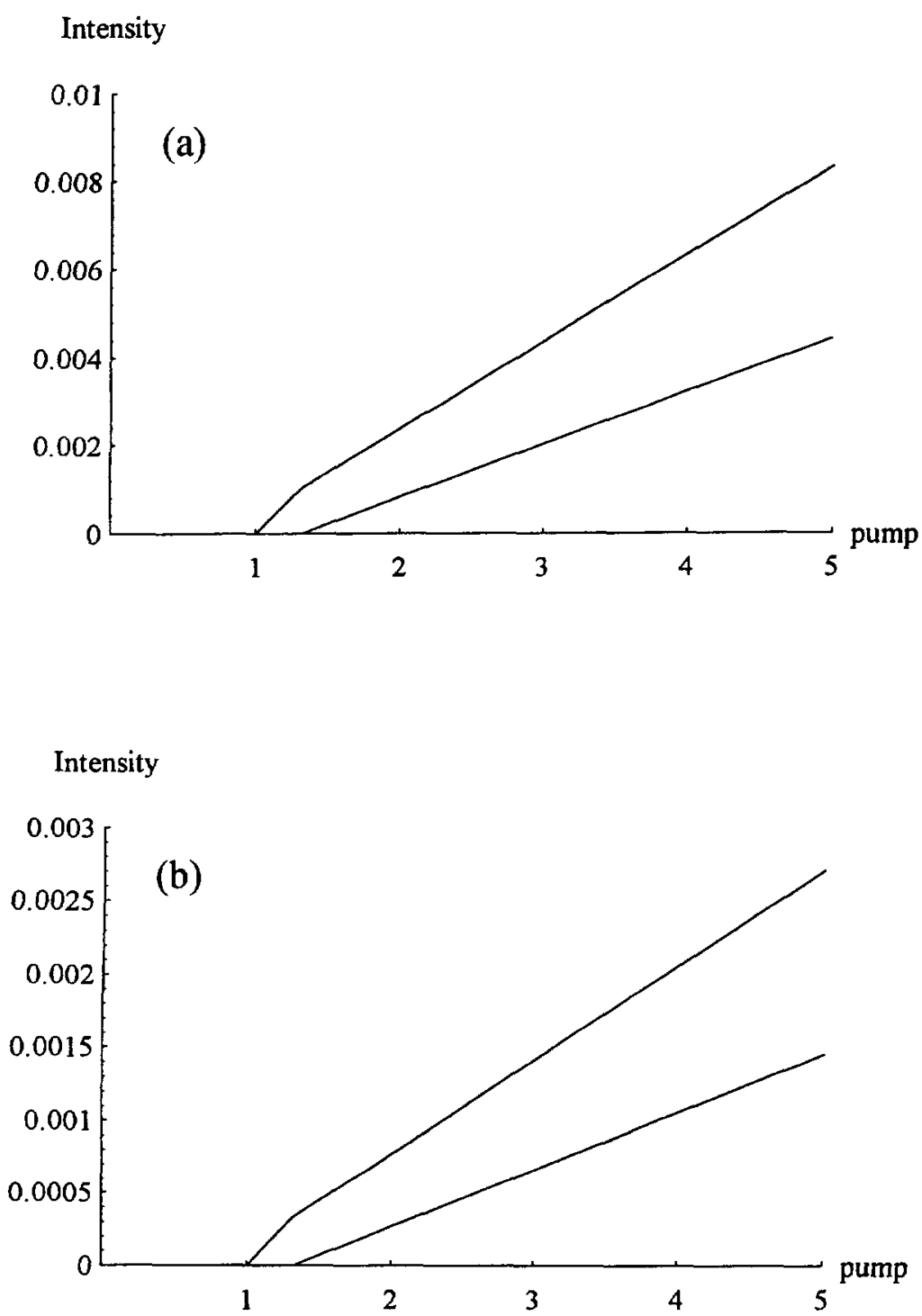

Fig. 2. - Evolution of the steady state intensities versus the pumping ratio: (a) $x=0$, (b) $x=0.10$. The upper and lower curves correspond respectively to $I_{1}$ and $I_{2}$. 
where

$$
L=\left(\begin{array}{ccc}
-a_{2}-(1+\beta) \bar{I} & (-1+\beta) \bar{i} & 0 \\
(-1+\beta) \bar{i} & -a_{2}-(1+\beta) \bar{I} & 0 \\
0 & 0 & -a_{2}-a_{22} / 2-3 y \bar{I} \\
0 & 0 & a_{2}-a_{22} / 2 \\
A(1+\beta)(1-2 x) \bar{I} / 2 & A(1-\beta)(1-2 x) \bar{i} / 2 & 0 \\
A(1+\beta)(1-2 x) \bar{i} / 2 & A(1-\beta)(1-2 x) \bar{I} / 2 & 0 \\
0 & -(1+\beta) \bar{D} & 0 \\
0 & 0 & (-1+\beta) \bar{D} \\
-a_{22} / 2 & y\left(2-3 a_{+}\right) & 0 \\
-a_{22} / 2-y \bar{I} & -y d_{-} & 0 \\
A x y \bar{I} & 0 & 0 \\
A x y \bar{i} & 0 & 0
\end{array}\right)
$$

The eigenvalues are calculated from the characteristic equation :

$$
\operatorname{det}(L-\lambda I)=0 .
$$

Among the six eigenvalues of (13), two $\left(\lambda_{1}, \lambda_{2}\right)$ always remain real negative, while the other four are two pairs of complex conjugates. One pair $\left(\lambda_{3}, \lambda_{3}^{*}\right)$ of complex conjugate eigenvalues has a negative real part while the other $\left(\lambda_{4}, \lambda_{4}^{*}\right)$ can have a positive real part depending on the ion pair concentration $x$. Figure 3 gives the evolution of $\operatorname{Re}\left(\lambda_{3,4}\right)$ and $\operatorname{Im}\left(\lambda_{3,4}\right)$ for $r$ varying between 1 to 10 and for several values of the ion pairs concentration. These results demonstrate that there exist two eigenfrequencies (due to the imaginary part of the eigenvalues). The higher frequency is responsible for the classical relaxation oscillations, while the lower is characteristic of a bimode system and is responsible for the antiphase effects. Note that the latter frequency appears when the second mode reaches its threshold. Moreover, one sees that for high pair concentrations, there exist $r$ values leading to $\operatorname{Re}(\lambda)>0$ implying that the steady state is no longer stable. As reported in [8], the $r$-interval for which the steady state is unstable broadens when $x$ increases.

\section{Numerical Calculation}

In this section, system (3) is numerically solved using a fifth order Runge-Kutta method with an adaptative integration step. We consider different cases of interest : the transient behavior leading to a c.w. state, the $n T$-periodic regimes and the chaotic regime. In fact, for a given intermediate ion pair concentration, the three dynamical behaviors can be obtained simply by decreasing the pumping ratio from an upper value which is high enough to allow a c.w. operation.

5.1. CW REgIME. - The c.w. regime can be achieved either with low ion pair concentration whatever $r$, or with intermediate $x$ values for pumping rates high enough to allow a saturation of the paired ions. The eigenfrequencies of the system can be visualized in the transient regime. Figure $4 \mathrm{a}$ shows the evolution of the intensities in the case $x=10 \%$ and for a pump- 

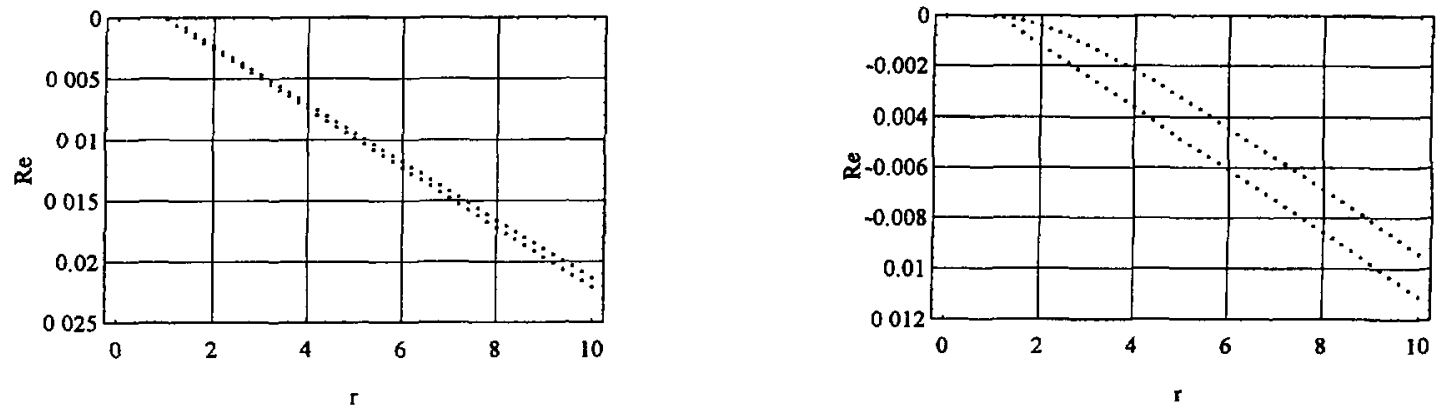

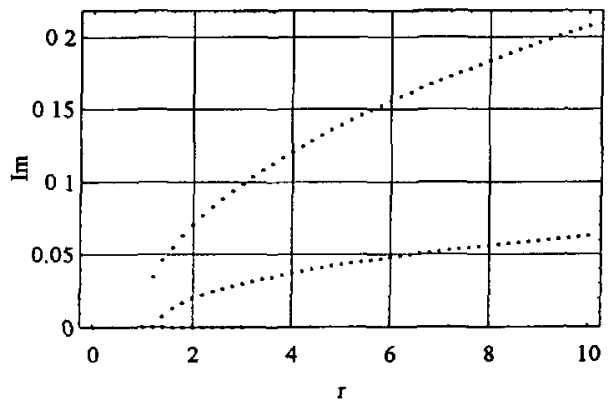

(a)
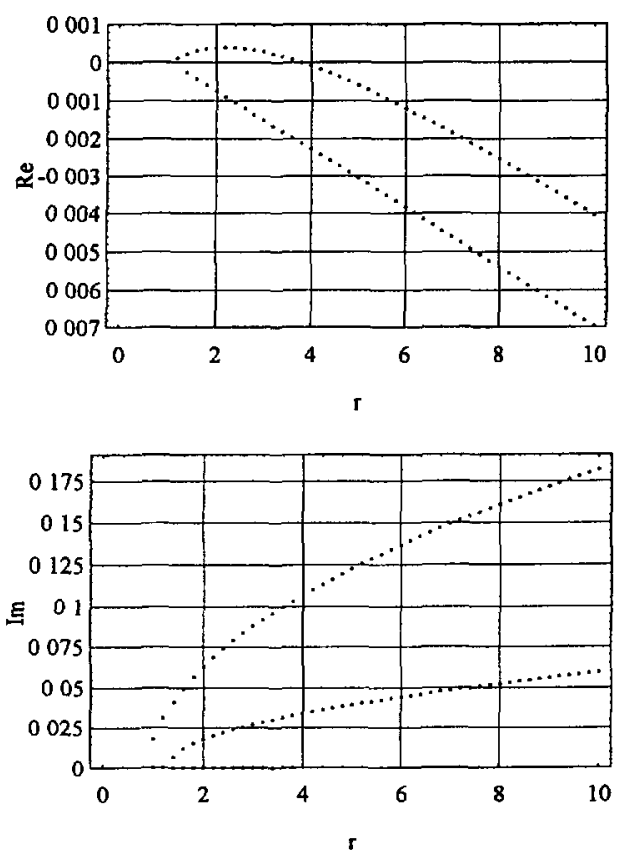

(c)

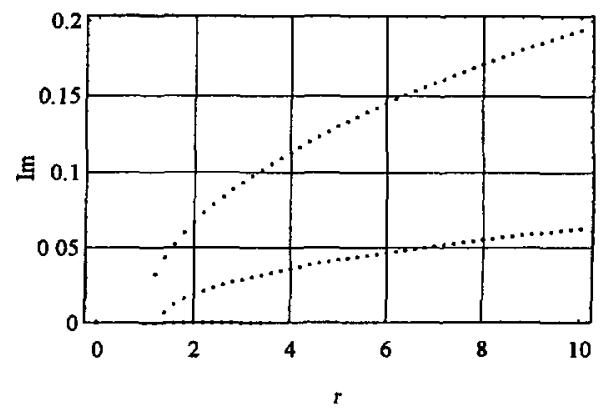

(b)
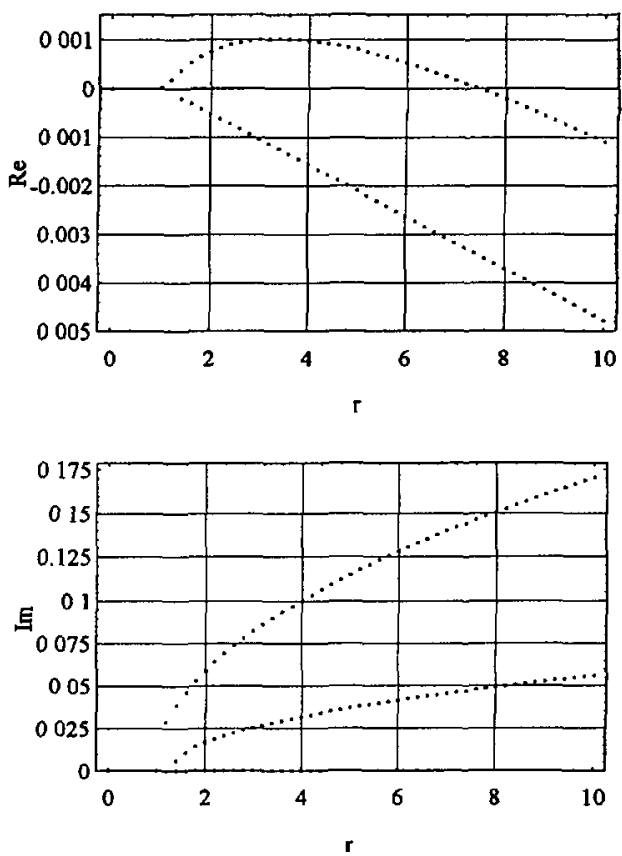

(d)

Fig. 3. - Evolution of the real and imaginary parts of the eigenvalues versus $r$ for several values of the ion pair concentration $x$. (a) $x=0 \%$, (b) $x=5 \%$, (c) $x=10 \%$ and (d) $x=15 \%$. The upper and lower curves correspond respectively to $\operatorname{Im}\left(\lambda_{4}\right)$ and $\operatorname{Im}\left(\lambda_{3}\right)$ for the imaginary part and to $\operatorname{Re}\left(\lambda_{4}\right)$ and $\operatorname{Re}\left(\lambda_{3}\right)$ for the real part of the eigenvalues. 
ing rate $r=4$. As expected from the results given by the linear stability analysis, together with the frequency of the relaxation oscillations $\left(f_{1}\right)$, there exists a lower frequency $\left(f_{2}\right)$ for which the two supermodes are in antiphase. Similar results are obtained for $x=5 \%$ and for any pumping ratio. Figure $4 \mathrm{~b}$ gives an example for $r=3$ where the two frequencies are clearly seen. Note that for $x=0$, the results are similar to that of reference [5] since system (3) reduces to a classical two-mode laser model.
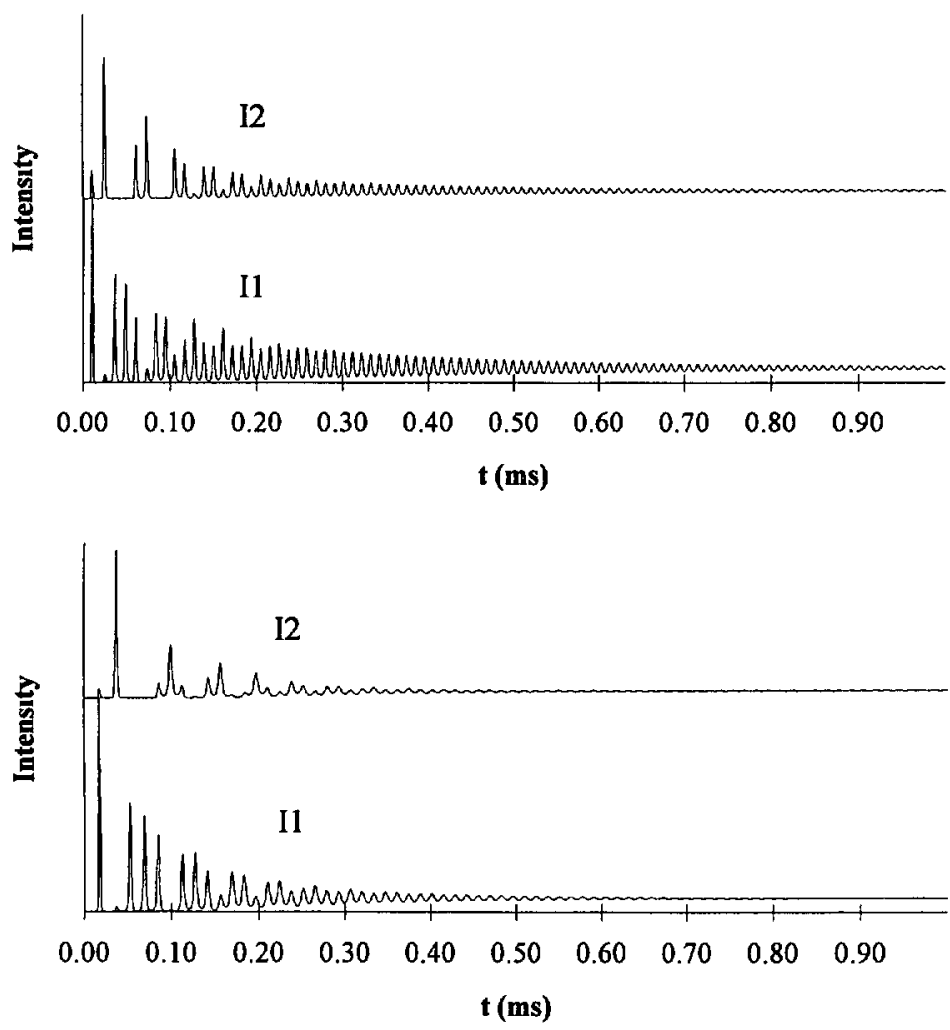

Fig. 4. - C.w. regime: (a) $x=10 \%$ and $r=4$; (b) $x=5 \%$ and $r=3$.

5.2. PERIODIC REGIMES. - The $n T$-periodic regimes appears for intermediate or high $x$-values in some range of pumping rates between the c.w. regime and the chaotic regime. The numerical simulations are given in Figure 5 which shows the time evolution of the two modes for different pumping rates and for $x=10 \%$. Figure 5a gives the transient regime for $r=3.4$ for which the system is $T$-periodic. As in the transient c.w. regime, the antiphase phenomenon is also present in the transient regime leading to the $T$-periodic orbit. When the pumping rate is decreased, the system becomes $2 T$-periodic as shown in Figure $5 \mathrm{~b}$ for $r=3$. The transition is continuous. A low frequency analysis of the time signals shows that in this case, there is a frequency locking of $f_{2}$ and $f_{1 / 2}$ [9]. While the pump is further decreased, the system changes suddenly from a $2 T$ - to a $3 T$-periodic regime as shown in Figure $5 \mathrm{c}$ for $x=10 \%$ and $r=2.3$. For the $3 T$ regime, a frequency locking of $f_{2}$ and $f_{1 / 3}$ occurs [9]. Antiphase effects occur in the asymptotic regimes for the $n T$-periodic orbits such as $n \geqslant 2$. Note however, that 
this phenomenon is not visible in the case of perfect symetry between the two modes (i.e. same pumping expressed by $\gamma=1$ ) where no $n T$-periodic orbits such as $n \geqslant 2$ have been obtained. It is worthwile to stress that the antiphase phenomena is due to the cross-saturation between the two modes and not to the asymetric pumping.
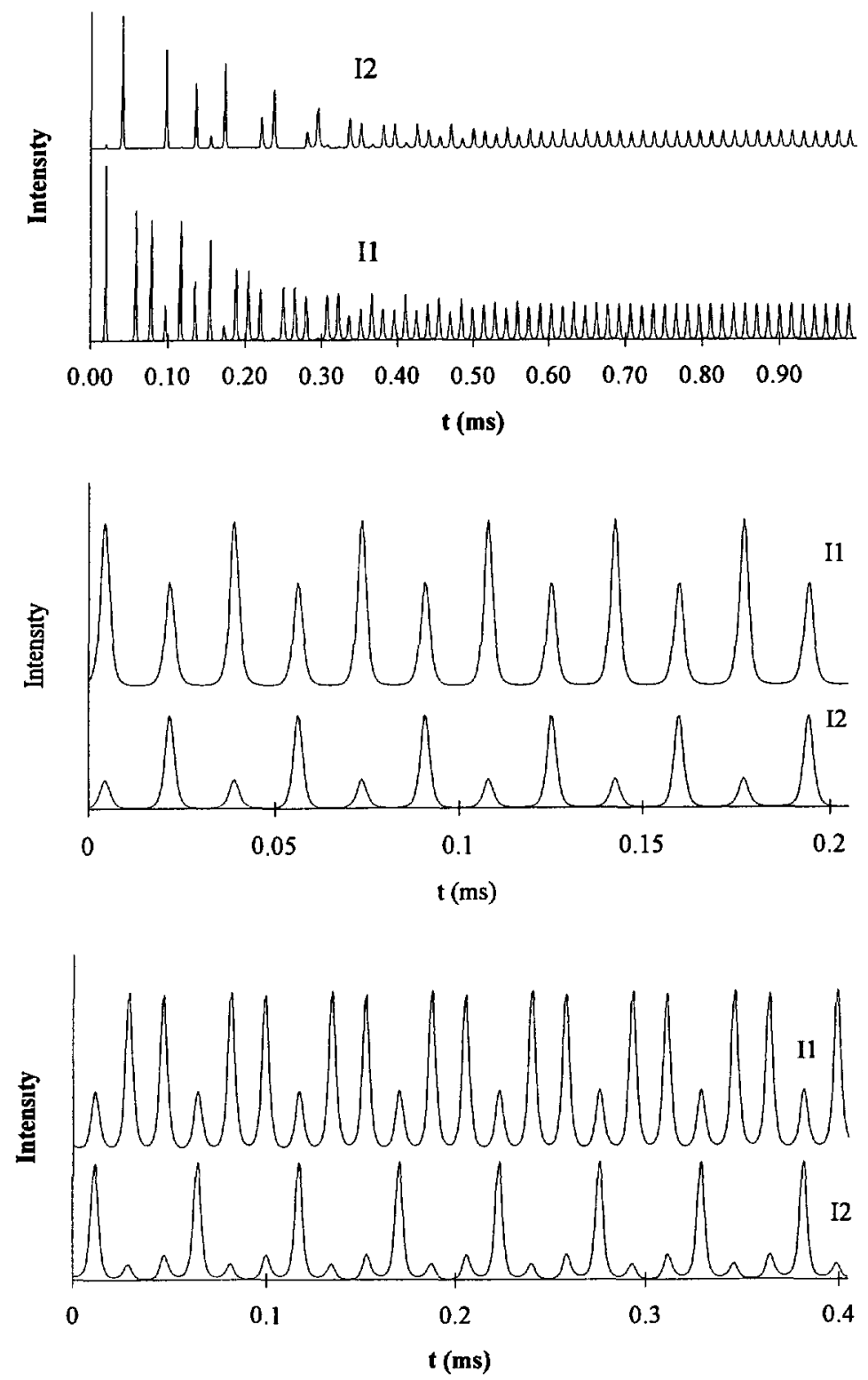

Fig. 5. - Self-pulsing regime: (a) $T$-periodic for $x=10 \%$ and $r=3.4$; (b) $2 T$-periodic for $x=10 \%$ and $r=3.0$, (c) $3 T$-periodic for $x=10 \%$ and $r=2.3$. 
5.3. ChaOTIC REGIME. - The chaotic regime [11] is achieved from the $3 T$-periodic regime by decreasing the pumping rate. The transition is sudden as we have experimentally reported in [9]. Figure 6 shows the chaotic behavior in the case of $x=10 \%$ and $r=2.2$. Similar results are obtained in the interval $r=1.6-r=2.25$. A first return map is given in Figure 7 corresponding to the time series of Figure 6. This map of two-mode chaos shows a two-dimensional distribution, suggesting that its strange attractor has a higher dimension than that of the single-mode chaos where the first return map is close to a one dimensional

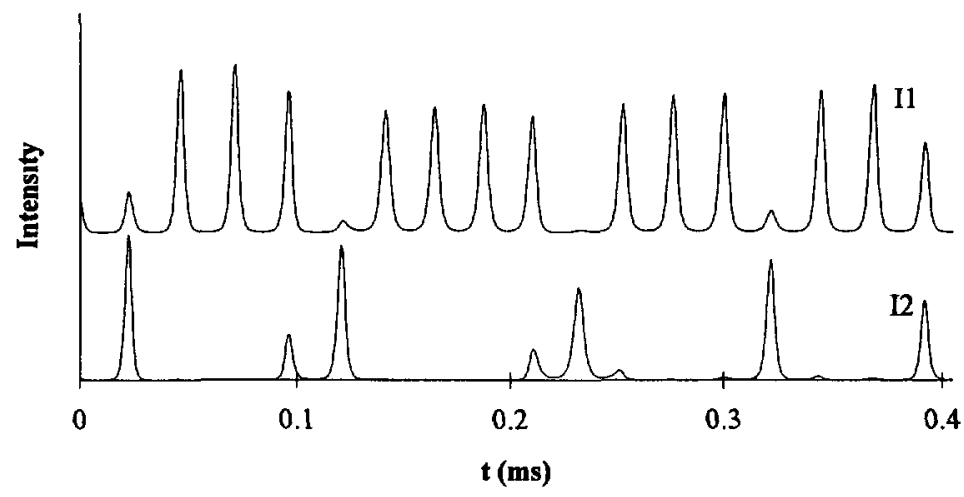

Fig. 6. - Chaotic regime for $x=10 \%$ and $r=2.2$.

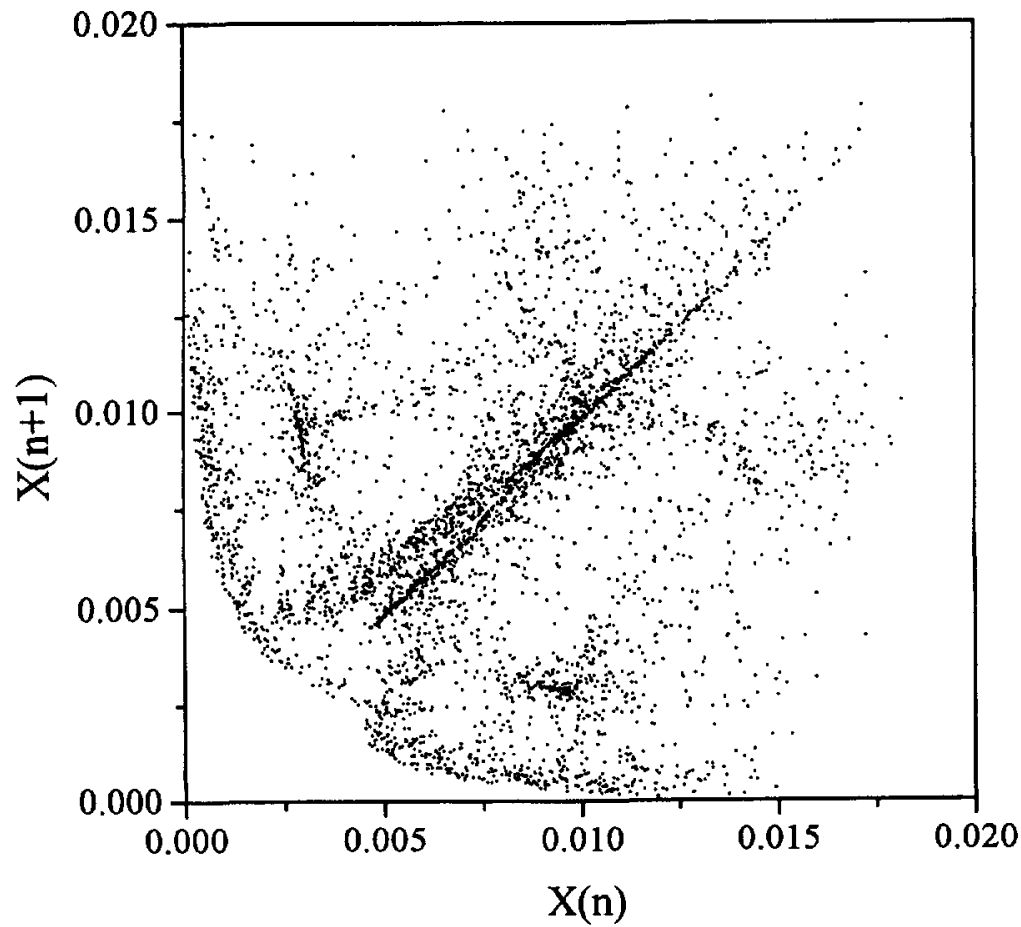

Fig. 7. - First return map in the chaotic regime corresponding to Figure 6. 
curve [12]. Figure 8 shows a representation of the strange attractor in the phase space defined by $D, d_{+}$and $I_{1}$. The attractor is distributed three-dimensionally. The calculated correlation dimension [13] of the attractor is about 2.8. This fractal dimension is compatible with that obtained experimentally which was found to be lower than 4.1 for any pumping rate [6].

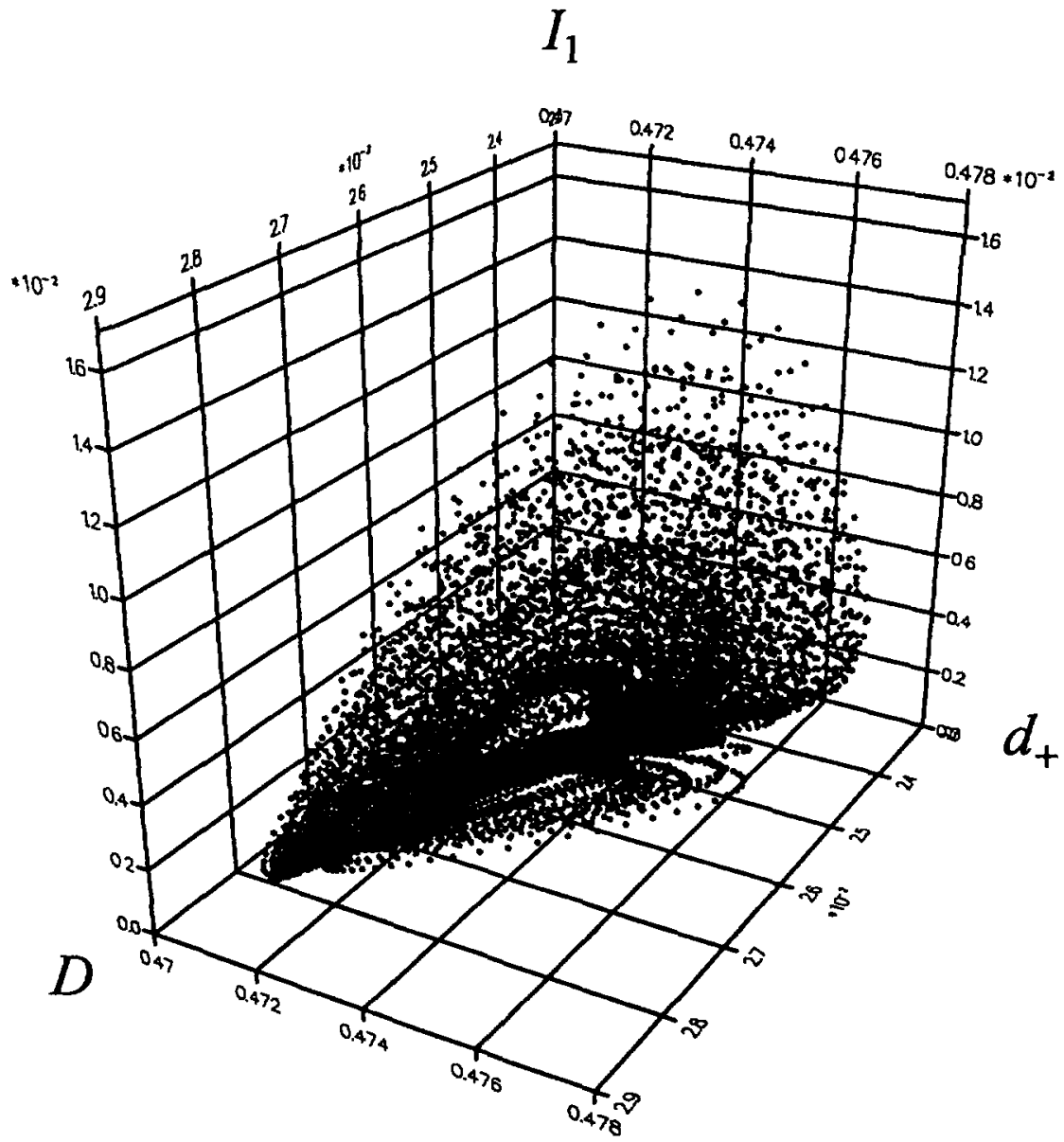

Fig. 8. - Portrait of the strange attractor in the phase space defined by $D, d_{+}$and $I_{1}$.

5.4. BifurCATION DiAgram. - It is convenient to represent the dynamical behavior of an EDFL in a bifurcation diagram which gives the maxima of the peak intensities when $r$ is varied. Figure 9 gives the evolution of the maxima of $I_{1}$ versus $r$. For high pumping rates $(r>3.75)$, the system is c.w. (in this case, we have plotted the steady state intensity). For slightly lower pumping ratios the laser becomes self-pulsing via a Hopf bifurcation [14]. For $r=3.3$, the system becomes $2 T$-periodic via a continuous transition. By further decreasing the pumping ratio, the laser suddenly becomes $3 T$-periodic for $r=2.75$. This behavior occurs until the pumping rate has reached the value $r=2.25$ for which the system is suddenly chaotic. A chaotic regime exists from this critical value to $r=1.6$. For this particular pumping 
rate the laser once again becomes $T$-periodic for the mode with lower losses ( $I_{1}$ in our case), the other mode does not lase because of the dichroism in the pumping process.

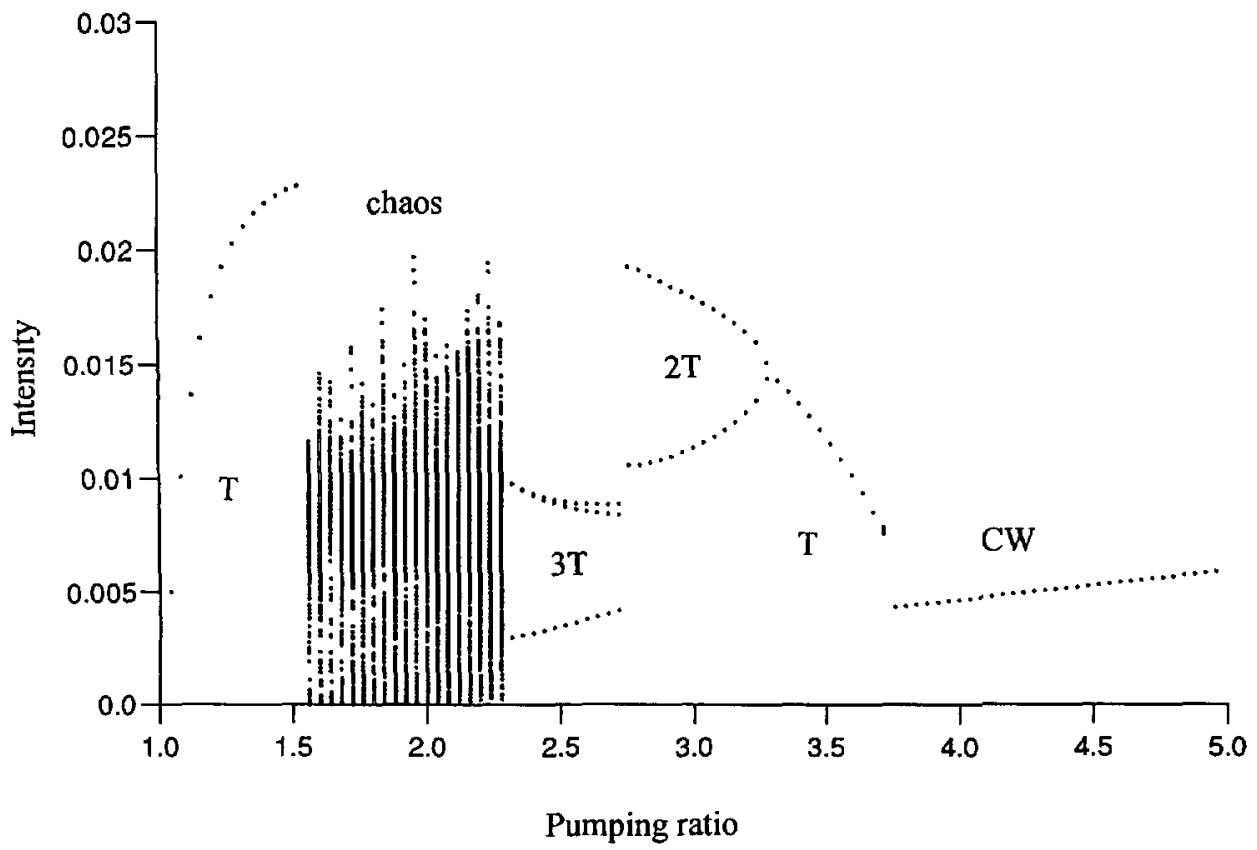

Fig. 9. - Bifurcation diagram for $I_{1}$.

\section{Conclusions}

This paper has been devoted to the theoretical modeling of erbium-doped fibre laser in a dual-wavelength operation. The model is based on the wellknown two-mode laser equations where we have added two equations for the population dynamics of ion pairs. The linear stability analysis has shown that a lower eigen-frequency appears together with the eigenfrequency responsible for the relaxation oscillations. Moreover, the presence of the ion pairs leads to unstable steady states. The numerical simulations for $x=10 \%$ has shown that, for a decreasing pumping rate, the dynamical scenario is : c.w. $\rightarrow T$-periodic $\rightarrow 2 T$-periodic $\rightarrow 3 T$ periodic $\rightarrow$ chaos $\rightarrow T$-periodic. Antiphase effects between the two modes has been obtained in the periodic and transient regimes. The chaotic regime has been characterized. In particular, the correlation dimension of the strange attractor has been found to be 2.8 .

A basic question remains concerning the identification of the route to chaos. We have numerically observed that the route to chaos is quasi-periodic for $x=12 \%:$ c.w. $\rightarrow T \rightarrow 2 T \rightarrow$ $4 T \rightarrow$ QP2 $\rightarrow$ chaos $\rightarrow 3 T \rightarrow$ chaos, the third frequency or QP3 is not observed in the scenario [11] as in the numerical experiment by Curry and Yorke [15]. The route to chaos $\mathrm{QP} 2 \rightarrow$ chaos $\rightarrow 3 T$ is similar to the one observed in the Rayleigh-Bénard instability for the B-structure [11]. The main differences are the appearence of two subharmonic bifurcations before QP2 $(2 T, 4 T)$. After QP2 the system experiences non-periodic motion with some sharp lines ( $3 T$ lines) (the dimension of the attractor ranges from 2 to 2.3). This transition from quasi-periodic motion to substructures indicates the progressive destruction of the two-torus 
towards a strange attractor. It has been mentionned [15] that the conversion of quasiperiodicity into chaos could occur from a two-torus if the three-torus is so unstable that the third uncommensurate frequency cannot be observed. In this respect, note that the QP2 regime is described in a narrow range of $r$ values. We could wonder wether this conjecture does not apply for the QP2 regime when $x$-values are lower as the route seems to $T \rightarrow 2 T \rightarrow 3 T \rightarrow$ chaos. Further investigations are needed to describe the lower $x$-values regimes.

The model is qualitatively in good agreement with our experimental results given in references [6] and [9] : it predicts the same route to chaos and also the antiphase effects and the frequency locking between the low frequency and a subharmonic of the high frequency. However, there is a systematic discrepancy between experiment and theory: the periods of the signals and the pumping rates at which the dynamics changes are found to be different. It is possible to match more closely the experimental results by a little change of the parameters. Nevertheless, for consistency, we have kept the same values that one used in the single-mode theory [8]. Note that the model developed in [8] leads only to the description of the self-pulsing effect, it does not lead to chaotic dynamics.

The model presented here could certainly be applied to describe the dynamics of the erbium-doped fibre laser operating on two polarizations with a suitable adaptation of the parameters.

The authors would like to thank the referees for their valuable comments to improve the manuscript.

\section{References}

[1] Otsuka K., Winner-takes-all dynamics and antiphase states in modulated multimode lasers, Phys. Rev. Lett. 67 (1991) 1090-1991.

[2] Wiesenfeld K., Bracikowski C., James G., Roy R., Observation of antiphase states in multimode laser, Phys. Rev. Lett. 65 (1990) 1749-1752.

[3] Bracikowski C., Roy R., Energy sharing in a chaotic multimode laser, Phys. Rev. A 43 (1991) 64556457.

[4] Le Boudec P., Le Flohic M., François P. L., Sanchez F., Stephan G., Self-pulsing in erbium-doped fibre laser, Opt. Quant. Electron. 25 (1993) 359-367.

[5] Bielawski S., Derozier D., Glorieux P., Antiphase dynamics and polarization effects in the Nd-doped fibre laser, Phys. Rev. A 46 (1992) 2811-2822.

[6] Le Boudec P., Sanchez F., Jaouen C., François P. L., Bayon J.-F., Stephan G., Antiphase dynamics and chaos in self-pulsing erbium-doped fibre laser, Opt. Lett. 18 (1993) 1890-1892.

[7] Le Boudec P., Sanchez F., François P. L., Delevaque E., Stephan G., Influence of ion pairs on the dynamical behavior of erbium-doped fibre lasers, Opt. Quant. Electron. 25 (1993) 501-507.

[8] Sanchez F., Le Boudec P., François P. L., Stephan G., Effects of ion pairs on the dynamics of erbium-doped fibre lasers, Phys. Rev. A 48 (1993) 2220-2229.

[9] Sanchez F., Le Flohic M., Stephan G., Le Boudec P., François P. L., Quasi-periodic route to chaos in erbium-doped fibre laser, accepted for publication to IEEE J. Quant. Electron. (3) (1995).

[10] Wolfram S., Mathematica, A system for doing mathematics by computer Second edition (AddisonWesley, Redwood, CA, 1991).

[11] Bergé P., Pomeau Y., Vidal C., Order within chaos, (Wiley, New York, 1984).

[12] Tanii K., Tachikawa M., Tohei T., Hong F.-L., Shimuzu T., Instability and chaos in two-mode oscillation of $\mathrm{CO}_{2}$ laser modulated by a saturable absorber, Phys. Rev. A 43 (1991) 1498-1501.

[13] Grassberger P., Procaccia I., On the characterization of strange attractors, Phys. Rev. Lett. 50 (1983) 346-350.

[14] Crawford J. D., Introduction to bifurcation theory, Rev. Mod. Phys. 63 (1991) 991-1037.

[15] Curry J. H. and Yorke J. A., A transition from Hopf bifurcation to chaos, Lect. Notes in Math. (Springer Verlag, New York, 1978), pp. 48. 